\title{
Sensor de conductividad eléctrica aparente para definir zonas homogéneas de manejo en suelos salinos
}

\section{Apparent electrical conductivity sensor used to define homogeneous management zones in saline soils}

\section{JULIO R. ROJAS B. ${ }^{1}$ \\ ANDRÉS F. GUERRA ${ }^{1}$ \\ JAIME A. ARÉVALO G. ${ }^{1}$ \\ LAUREANO GUERRERO J. ${ }^{2}$ \\ FABIO R. LEIVA ${ }^{3,4}$}

Toma de datos prelabranza en un lote para incorporación a la agricultura.

Foto: J.R. Rojas B.

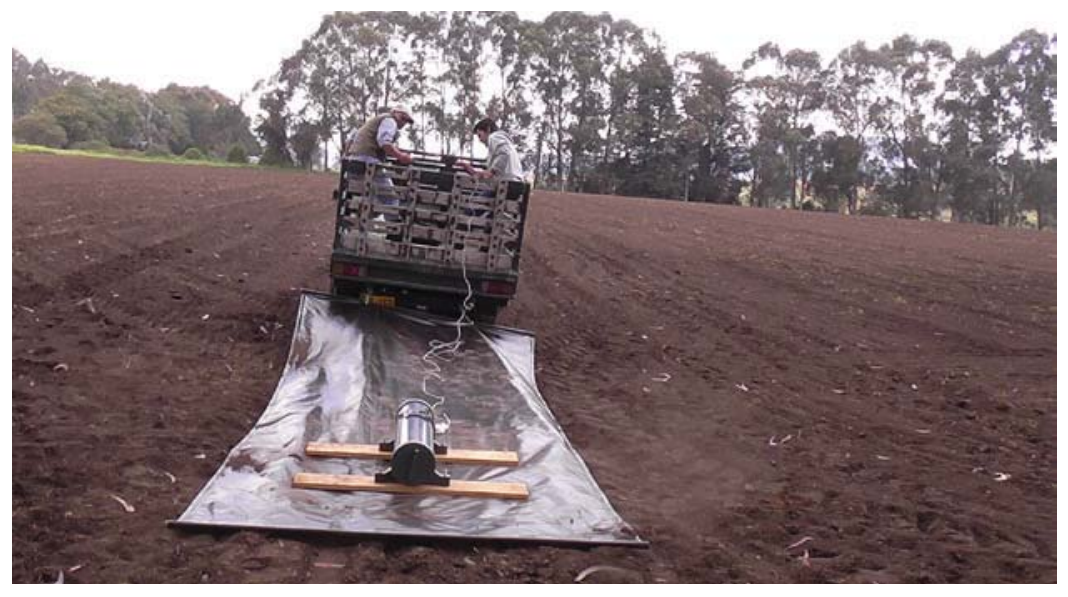

\section{RESUMEN}

La degradación de suelos por sales es un problema grave a nivel mundial. En Colombia, aproximadamente el $5 \%$ de los suelos sufren procesos de salinización, con impactos negativos en servicios ecosistémicos. Identificar el tipo y estado de salinidad de suelos agrícolas resulta complejo y requiere considerar la variabilidad que ocurre al interior de un lote destinado a la producción. Esto se puede realizar identificando zonas homogéneas de manejo (ZM) según las condiciones específicas del suelo. Este artículo presenta avances de una investigación en la cual se usa un sensor de conductividad eléctrica aparente (CEa) para identificar ZM en suelos salinos. Los ensayos de campo se realizaron en dos lotes agrícolas del Centro de Investigación Agropecuaria Marengo (Mosquera, Cundinamarca). Se usó un sensor Geonics ${ }^{\circledR}$ EM38-MK2 para tomar datos de CEa en transectos paralelos espaciados entre si $15 \mathrm{~m}$, con una profundidad de exploración de $0,75 \mathrm{~m}$. Simultáneamente, se tomaron datos de contenido de agua en el suelo (Wsc), en una grilla $15 \times 50 \mathrm{~m}$, mediante el sensor Time Domain. El análisis de datos incluyó estadística descriptiva con SPSS V22 y elaboración de mapas de CEa y de Wsc en un Sistema de Información Geográfico usando ArcGIS V10; esto permitió identificar 3 ZM. En cada zona se realizaron tres muestreos para análisis en laboratorio de propiedades fisicoquímicas de suelos. El estudio mostró importantes ventajas del sensor EM38-MK2 para identificar diferentes ZM caracterizadas por las propiedades químicas del suelo asociadas a la salinidad. La metodología usada hace un aporte significativo al manejo por sitio-específico de suelos salinos en la agricultura.

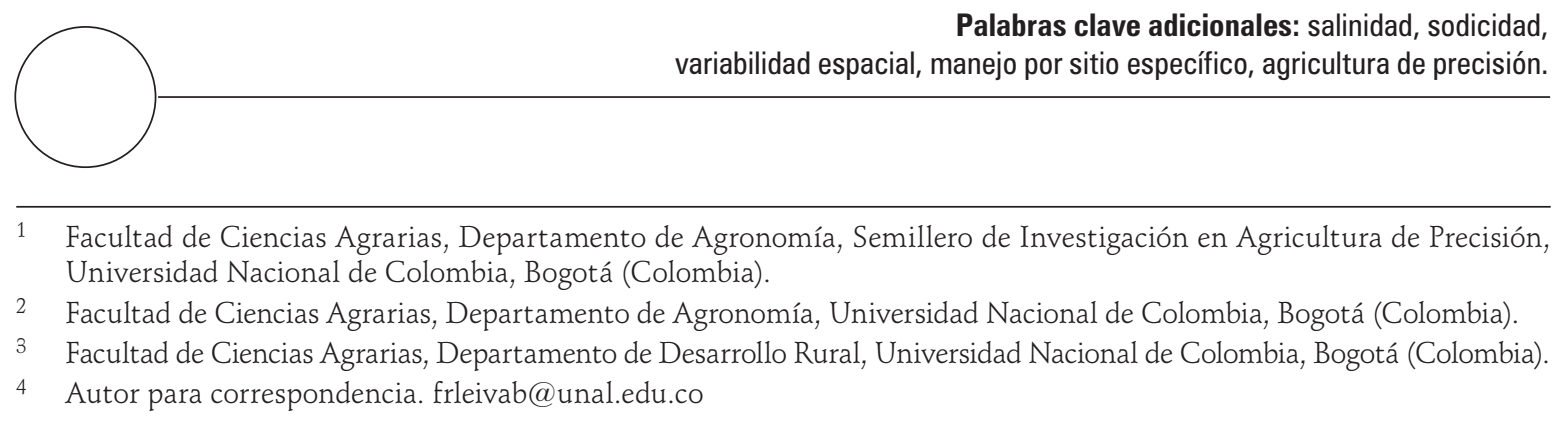




\section{ABSTRACT}

Salt-induced soil degradation is a serious problem worldwide. In Colombia, about $5 \%$ of soils are affected by salinization processes, with negative impacts on ecosystem services. The identification of the type and state of salinity in agricultural soils is complex and requires consideration of the variability that occurs within the crop field. This can be done by identifying homogeneous management zones (MZ) according to site-specific soil conditions. This paper presents research advances in which a soil apparent electrical conductivity sensor (ECa) was used to identify the MZ in saline soils. Field trials were conducted in two agricultural fields at the Marengo Agricultural Research Center (Mosquera, Cundinamerca). An EM38-MK2 Geonics ${ }^{\circledR}$ sensor was used to record CEa data in parallel transects spaced $15 \mathrm{~m}$ apart, with an effective exploration depth of $075 \mathrm{~m}$. Simultaneously, the soil water content (Wsc) was measured in a regular $15 \times 50 \mathrm{~m}$ grid using a Time Domain Reflectometer. The data analysis included descriptive statistics with SPSS 22 and mapping of the ECa and Wsc using ArcGIS 10; this allowed for the identification of three MZs per field. In each MZ, three soil samples were taken for laboratory analysis of the physicochemical properties of the soil. The study showed significant advantages when using the EM38-MK2 to identify MZs when characterized by differences in the soil chemical properties associated with salinity. The employed methodology will make a significant contribution to sitespecific management of saline soils for agriculture.

Additional key words: salinity, sodicity, spatial variability, site-specific management, precision farming.
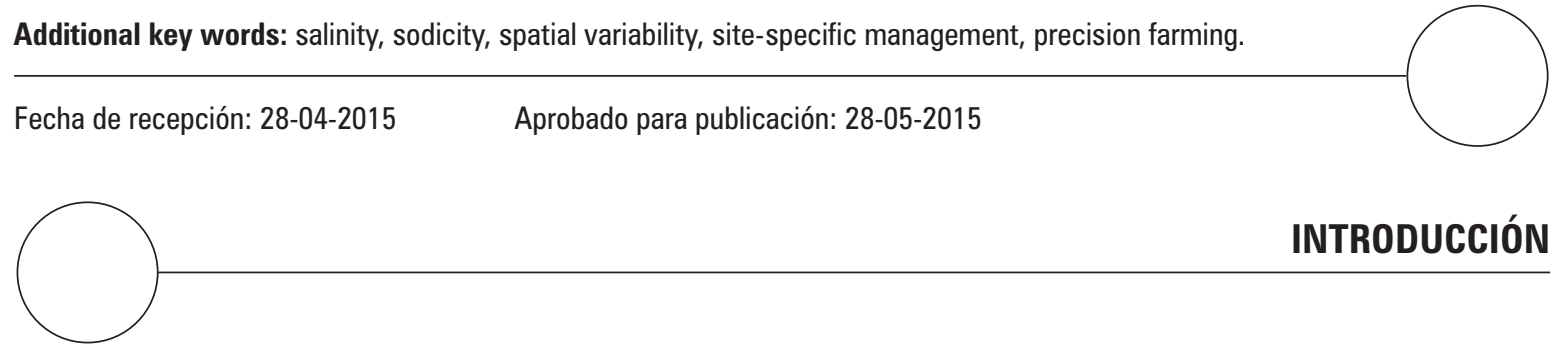

INTRODUCCIÓN

La degradación de suelos por sales es un problema que afecta alrededor de 1 billón de hectáreas a nivel mundial (Pla, 2014). En Colombia, el Ministerio de Ambiente y Desarrollo Sostenible estimó que alrededor del $5 \%$ de los suelos se ven afectados por procesos de salinización, con riesgo de desertificación en regiones áridas y semiáridas, y afectación de servicios ecosistémicos claves para la sociedad, tales como la producción de alimentos, fibras y maderas, los recursos genéticos y el ciclo del agua (MADS, 2013).

La salinización de suelos agrícolas está relacionada con procesos hidrológicos de evapotranspiración y drenaje, y puede ocurrir en condiciones de secano o regadío (Pla, 2014). En la agricultura moderna, la salinización se asocia principalmente al riego, particularmente en suelos mal drenados o con niveles freáticos cercanos a la superficie, cuando se usan aguas con iones tales como $\mathrm{Ca}^{2+}, \mathrm{Na}^{+}, \mathrm{CO}^{3-}, \mathrm{HCO}^{3-}, \mathrm{Cl}^{-}, \mathrm{SO}_{4}^{2-}$ y nutrientes como N, P, K y B (Ayers y Westcot, 1994). La acumulación de sales aumenta el potencial osmótico del suelo afectando negativamente los cultivos, puesto que se restringe la toma de agua por parte de las raíces, particularmente en suelos de texturas finas, y además, algunos iones son en sí mismos fitotóxicos (Munns, 2009). De otro lado, la presencia de $\mathrm{Na}^{+}$intercambiable puede causar dispersión de la arcilla haciendo inestable la estructura del suelo y aumentar el pH de este, lo cual restringe la disponibilidad de algunos nutrientes para el cultivo (FAO, 1988).

Los suelos salinos se caracterizan por el predominio de sales solubles neutras de cloruros y sulfatos de $\mathrm{Na}$, Ca y $\mathrm{Mg}$, tienen un $\mathrm{pH}<8,2$, conductividad eléctrica en pasta saturada (CE) $>4 \mathrm{dS} \mathrm{m} \mathrm{m}^{-1}$ a $25^{\circ} \mathrm{C}$, relación de absorción de $\mathrm{Na}$ (RAS) $<13$ y porcentaje de $\mathrm{Na}$ intercambiable (PSI) $<15$, además usualmente presentan cantidades apreciables de Ca y Mg. Por su parte, los suelos sódicos presentan cantidades considerables de sales de $\mathrm{Na}$ (ej. $\mathrm{Na}_{2} \mathrm{CO}_{3}$ ) capaces de sufrir 
hidrólisis alcalina, tienen un $\mathrm{pH}>8,2$, porcentaje de $\mathrm{Na}$ intercambiable (PSI) $\geq 15$, RAS $\geq 13$ y generalmente $\mathrm{CE} \geq 4 \mathrm{dS} \mathrm{m} \mathrm{m}^{-1}$ a $25^{\circ} \mathrm{C}$, aunque puede ser superior en presencia de altos contenidos de $\mathrm{Na}_{2} \mathrm{CO}_{3}$; en estos suelos comúnmente el $\mathrm{Ca}$ y el $\mathrm{Mg}$ se encuentran en pequeñas cantidades debido a que el $\mathrm{pH}$ tiende a precipitar estos elementos. De otro lado, los suelos salino-sódicos combinan características de los dos anteriores (FAO, 1988; Soil Survey Staff, 1993).

El manejo de suelos agrícolas afectados por sales requiere una clara identificación del problema y sus causas, e incluye evaluaciones de campo y de laboratorio (Ayers y Westcot, 1994). La gestión específica depende de las sales presentes y comprende decisiones acerca del tipo de cultivo (baja susceptibilidad a sales), así como del manejo del agua y del suelo. Para suelos salinos, se recomienda el "lavado" usando agua con bajo contenido de sales para arrastrar aquellas presentes en el suelo hacia capas profundas en el perfil o retirarlas del lote. Los suelos sódicos son difíciles de manejar; en estos se debe mejorar el drenaje, realizar operaciones de labranza para diluir capas con altos contenidos de $\mathrm{Na}$ e incorporar enmiendas tales como yeso $\left(\mathrm{CaSO}_{4}\right)$ y S (Horneck et al., 2007). Por su parte, aplicaciones de abonos orgánicos en suelos arenosos permiten mejorar la capacidad del almacenamiento de agua, retener nutrientes y mitigar los efectos negativos de usar aguas salinas, mientras que en suelos pesados esos abonos contribuyen a mejorar su estructura y drenaje, facilitando el lavado de sales (Mushtaque et al., 2013).

La identificación del tipo y estado de salinidad de un suelo agrícola resulta compleja, puesto que exige entender los procesos pedogenéticos y las prácticas de manejo que lo han llevado a esa condición (Athar y Ashraf, 2009). Adicionalmente, requiere considerar la variabilidad que se da en el interior de un lote agrícola en términos de microtopografía y de propiedades del suelo (Burrough, 1993), y cómo estas se expresan en condiciones de salinidad (Doolittle y Brevik, 2014). Además, existe heterogeneidad en el rendimiento de los cultivos como respuesta a la interacción de factores biológicos, edáficos, topográficos, climáticos y antropogénicos (Mulla y Schepers, 1997). Esto plantea la necesidad de manejos sitio-específicos, identificando zonas homogéneas de manejo (ZM) de acuerdo con las condiciones particulares del suelo y del cultivo, orientados a mejorar el uso de insumos y la eficiencia productiva (Pautasso et al., 2010; De Benedetto et al., 2013).

La conductividad eléctrica aparente del suelo (CEa), entendida como aquella que se mide insitu en el suelo sin disturbarlo, permite identificar ZM a partir de la salinidad, contenido de agua (Wsc), textura, capacidad de intercambio catiónico (CIC), materia orgánica y densidad aparente del suelo (Corwin y Lesch, 2005; Sudduth et al., 1997). La metodología incluye el uso de sensores de inducción electromagnética o de resistividad eléctrica que fueron usados inicialmente en la identificación de suelos afectados por sales (Corwin y Rhoades, 1982). Así, la evaluación de la variabilidad de la CEa permite detectar problemas de salinidad e identificar ZM para definir prácticas específicas de manejo (Corwin y Plant, 2005).

El objetivo de este artículo es presentar avances de una investigación en la cual se usa un sensor de CEa para identificar ZM en suelos salinos.

\section{MATERIALES Y MÉTODOS}

\section{Ubicación}

El ensayo se realizó durante el segundo semestre de 2014 en dos lotes dedicados a cultivos transitorios, lote 1 (1,1 ha) y lote 2 (1,4 ha), ubicados en el Centro de Investigación Agropecuaria Marengo (CIAM) en Mosquera (Cundinamarca). Los suelos fueron clasificados como Cumulic haplustolls y Aquandic humaquepts (tabla 1). 
Tabla 1. Clasificación taxonómica de los suelos para los dos lotes evaluados.

\begin{tabular}{|c|c|c|c|} 
Lote & $\begin{array}{c}\text { Unidad } \\
\text { cartográfica }\end{array}$ & \multicolumn{2}{c|}{ Unidad taxonómica de suelo } \\
\hline 1 & Consociación & $\begin{array}{l}\text { Cumulic haplustoll, franca fina, mezclicas } \\
\text { semi-activa, isomésica }\end{array}$ & $\begin{array}{l}\text { Suelos profundos, bien drenados, altos } \\
\text { contenidos de sales y sodio, fertilidad alta }\end{array}$ \\
\hline 2 & Consociación & $\begin{array}{l}\text { Aquandic humaquepts, arcillosa sobre medial, } \\
\text { mezclada, activa, isomésica }\end{array}$ & $\begin{array}{l}\text { Suelos superficiales, pobremente drenados, } \\
\text { capas con alta resistencia a la penetración, } \\
\text { fuertemente ácido, fertilidad alta }\end{array}$ \\
\hline
\end{tabular}

Fuente: adaptada de IGAC (2014).

\section{Muestreo y mapeo de la conductividad eléctrica aparente (CEa)}

Con el fin de identificar zonas homogéneas de manejo, previo a la fertilización y labranza del suelo, se midió la CEa con un sensor Geonics ${ }^{\circledR}$ EM38-MK2, enganchado a un tractor John Deere 5400 DT. El sensor se usó con el dipolo vertical y un espaciamiento entre bobinas de $0,5 \mathrm{~m}$ (alcance hasta una profundidad en el suelo de $0,75 \mathrm{~m}$ ), mediante transectos paralelos separados $15 \mathrm{~m}$ entre sí. Simultáneamente se midió el contenido de agua en el suelo (Wsc) con el medidor FieldScout TDR 300 (Spectrum Technologies, Aurora, IL), usando una grilla de $15 \times 50$ $\mathrm{m}$. Considerando la alta sensibilidad del sensor EM38-MK2, ya que realiza hasta 21 medidas de CEa por segundo, se filtraron los datos para eliminar valores atípicos. Los datos de CEa y de Wsc se analizaron con técnicas de estadística descriptiva usando el programa IBM SPSS Statistics ${ }^{\circledR}$ versión 22 (IBM, 2013). Posteriormente, se evaluó la variabilidad espacial en terreno usando un Sistema de Información Geográfica y técnicas de kriging ordinario, con la herramienta ArcGIS 10 (ESRI, 2011). Para el análisis estructural de CEa y Wsc, se seleccionó el semivariograma de mejor ajuste mediante validación cruzada y los siguientes criterios para el error de predicción: i) error medio estandarizado cercano a 0 , ii) error cuadrático medio pequeño, iii) error estándar promedio similar al error cuadrático medio, iv) error cuadrático medio estandarizado cercano a 1. A partir del semivariograma de mejor ajuste, se aplicó kriging ordinario, evaluando posible anisotropía. La confiabilidad de los mapas generados se comprobó con el mapa de errores, verificando que el error máximo fuera del 10\%. De manera similar a lo reportado por Simón et al. (2013), en cada uno de los lotes se definieron tres zonas de manejo, usando cuantiles como método de clasificación (ESRI, 2011). Las zonas identificadas se denominaron "baja", "media" y "alta" de acuerdo con los valores encontrados de CEa.

En cada una de las zonas identificadas se definieron tres puntos de muestreo en los cuales se tomaron muestras de suelo para análisis químico en laboratorio de: $\mathrm{pH}$ (prueba de suspensión en agua 1:1 y uso del potenciómetro), CIC (valoración volumétrica acetato- $\mathrm{NH}_{4} 1 \mathrm{M}, \mathrm{pH} 7$ y $\mathrm{NaCl}$ ), contenido de $\mathrm{Na}$, Ca y $\mathrm{Mg}$ (acetato- $\mathrm{NH}_{4}$ $1 \mathrm{M} \mathrm{pH}$ 7), cloruros y sulfatos (en pasta saturada) y textura (arcilla, limo y arena por el método de Bouyucos). A estos resultados se les aplicó estadística descriptiva. Según el coeficiente de variación (CV), se evaluó la variabilidad de las propiedades de suelo ajustando la escala propuesta por Larreal (2005): muy baja variabilidad de 0 a $<15 \%$, baja variabilidad de $\geq 15$ a $<30 \%$, moderada variabilidad de $\geq 30$ a $<45 \%$, variabilidad alta de $\geq 45$ a $<60 \%$ y muy alta variabilidad $\geq 60 \%$. Luego se realizó una comparación de medias entre los dos lotes para las diferentes propiedades evaluadas, y en cada uno de los lotes se realizó un análisis de varianza y de diferencia 
mínima significativas (DMS) entre zonas usando el programa estadístico SPSS V22.

\section{RESULTADOS Y DISCUSIÓN}

La estadística descriptiva para CEa y Wsc en los dos lotes muestra distribuciones razonablemente normales (considerando asimetría y curtosis), excepto para Wsc en el lote 2 (tabla 2). La variabilidad para estas propiedades resultó de baja a moderada ( $\geq 15 \%$ CV <45\%) (Larreal, 2005), lo cual sugiere evaluar una posible zonificación para el manejo en esos lotes.

En el análisis estructural de CEa y Wsc se seleccionaron modelos de semivariograma Stable para el lote 1 y gaussiano para el lote 2 (ArcGIS), según la validación cruzada y los errores de predicción (tabla 3). Para CEa se utilizó anisotropía con ángulos de $41,5^{\circ}$ y $73,2^{\circ}$ respectivamente en los lotes 1 y 2 , puesto que bajo esta condición se mejoraron los errores de predicción. A partir de esos semivariogramas, se obtuvieron los mapas correspondientes mediante kriging ordinario (figura 1).

Considerando cierta similitud de los mapas de CEa y de Wsc en los dos lotes estudiados, se evaluó el uso de cokriging. Sin embargo, los errores de la validación cruzada no mejoraron, indicando escasa correlación espacial entre esas propiedades, probablemente debido a la presencia de sales que conlleva a que otras propiedades del suelo tengan menor incidencia en los valores de CEa (Corwin et al., 2012). En consecuencia, se dejó el mapa originalmente generado para la CEa, con tres ZM que se clasificaron como "baja", "media" y "alta" según los valores de esta propiedad edáfica.

\section{Salinidad y otras propiedades fisicoquímicas de los lotes}

La variabilidad del pH resultó muy baja en cada uno de los lotes (CV <15\%), con diferencias altamente significativas (1\%) entre estos (tabla 4). Así, el lote 1 se clasifica como moderadamente ácido $(5,6-6,0)$ y el lote 2 , como fuertemente ácido $(5,1-5,5)$. Por su parte, CE presentó variabilidad alta en el lote 1 y moderada en el lote 2 , con valores superiores (altamente significativos) en el lote con menor acidez (lote 1), que superan el umbral para suelos sódicos $(>4$ dS $\mathrm{m}^{-1}$ ) (FAO, 1988; Soil Survey Staff, 1993). De otro lado, los contenidos de $\mathrm{Ca}$ y de $\mathrm{Mg}$ resultaron altos (Ca $>6 \mathrm{meq} \mathrm{L}^{-1}$ y Mg $\left.>1,8 \mathrm{meq} \mathrm{L}^{-1}\right)$, con relaciones $\mathrm{Ca} / \mathrm{Mg}$ cercanas a 1 que indican deficiencia de Ca (Castro y Gómez, 2010), pero en el lote 1 los valores promedio de estas propiedades (con muy alta variabilidad) fueron superiores con diferencias significativas respecto al segundo lote.

La historia de manejo de esos lotes indica prácticas inadecuadas de fertilización que pueden conducir a los desbalances citados (Zhaoyong et al., 2014) y posibles efectos del agua de riego, cuya

Tabla 2. Estadística descriptiva para conductividad eléctrica aparente (CEa) y contenido de agua en el suelo (Wsc) en los dos lotes evaluados.

\begin{tabular}{|c|c|r|r|r|r|r|r|r|}
\hline Propiedad & Lote & Media & Mínimo & Máximo & $\begin{array}{c}\text { Desviación } \\
\text { estándar }\end{array}$ & Asimetría & Curtosis & CV (\%) \\
\hline \multirow{2}{*}{ CEa (mS/m) } & 1 & 203,8 & 91,2 & 385,9 & 76,97 & 0,53 & $-0,83$ & 37,77 \\
\cline { 2 - 9 } & 2 & 96,8 & 73,7 & 151,1 & 17,02 & 1,17 & 0,77 & 17,58 \\
\hline \multirow{2}{*}{ Wsc (\%) } & 1 & 54,3 & 18,2 & 64,3 & 13,58 & $-0,10$ & 0,75 & 25,03 \\
\cline { 2 - 9 } & 2 & 42,7 & 17,0 & 42,3 & 15,89 & $-1,32$ & $-1,42$ & 37,16 \\
\hline
\end{tabular}

CV: Coeficiente de variación. 


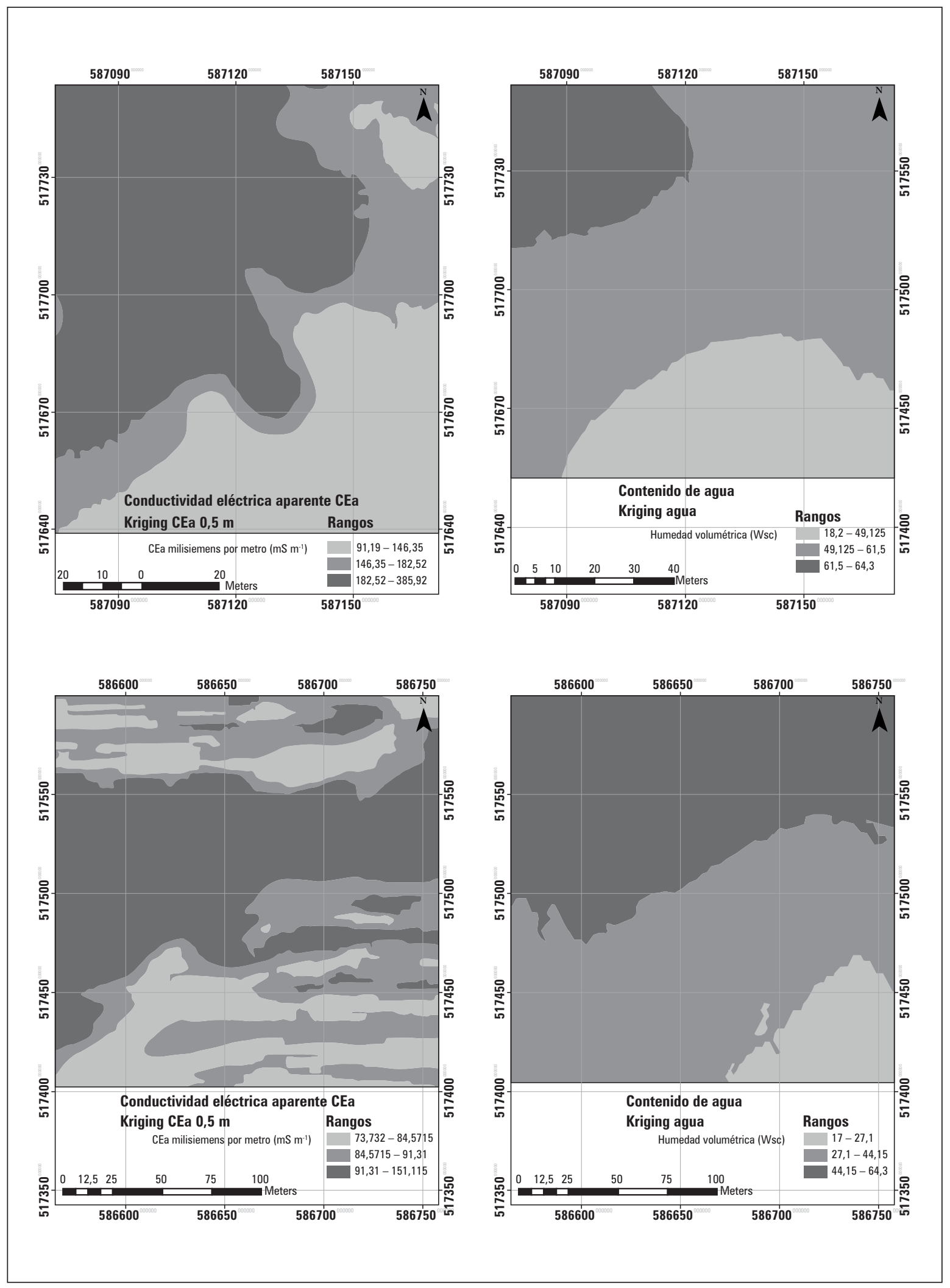

Figura 1. Mapas de conductividad eléctrica aparente (CEa), medidos en $\mathrm{mS} \mathrm{m}^{-1}$ a $0,5 \mathrm{~m}$ de profundidad y de contenido de agua en el suelo (Wsc) para los lotes 1 (arriba) y 2 (abajo). 
Tabla 3. Parámetros de los semivariogramas usados para el análisis estructural de conductividad eléctrica aparente (CEa) y de contenido de agua en el suelo (Wsc) en los dos lotes evaluados.

\begin{tabular}{|l|c|l|r|r|r|r|r|r|c|}
\multicolumn{1}{|c|}{ Propiedad } & Lote & Modelo & \multicolumn{1}{|c|}{ Pepita } & Meseta & \multicolumn{1}{|c|}{ Rango } & EME & ECM & EEP & ECME \\
\hline \multirow{2}{*}{ CEa } & 1 & Stable & 0 & 8514,52 & 67,86 & 0,0033 & 6,4893 & 6,8322 & 0,9934 \\
\cline { 2 - 11 } & 2 & Gaussiano & 6,41 & 351,15 & 78,08 & 0,0021 & 2,8788 & 2,8626 & 1,0096 \\
\hline \multirow{2}{*}{ Wsc } & 1 & Stable & 134,02 & 255,69 & 110,81 & 0,0082 & 12,3469 & 12,6161 & 0,9813 \\
\cline { 2 - 11 } & 2 & Gaussiano & 209,33 & 271.29 & 83,10 & $-0,0008$ & 15,0850 & 16,0931 & 0,9414 \\
\hline
\end{tabular}

CEa: conductividad eléctrica aparente; Wsc: contenido de agua en el suelo; EME: error medio estandarizado; ECM: error cuadrático medio; EEP: error estándar promedio; ECME: error cuadrático medio estandarizado.

Tabla 4. Resultados de estadística descriptiva para sales y otras propiedades fisicoquímicas de los dos lotes evaluados.

\begin{tabular}{|c|c|c|c|c|c|c|c|c|}
\hline $\begin{array}{l}\text { Propiedad/ } \\
\text { Unidad }\end{array}$ & Lote & Media & Mín. & Máx. & $\begin{array}{c}\text { Desviación } \\
\text { estándar }\end{array}$ & Sesgo & Curtosis & CV (\%) \\
\hline \multirow{2}{*}{$\mathrm{pH}$} & 1 & $5,66^{* *}$ & 5,39 & 6,01 & 0,21 & 0,20 & $-0,72$ & 3,71 \\
\hline & 2 & 5,16 & 4,85 & 5,54 & 0,20 & 0,49 & 0,75 & 3,88 \\
\hline \multirow{2}{*}{$\begin{array}{l}\text { CIC (meq por } \\
\text { cada } 100 \mathrm{~g} \text { ) }\end{array}$} & 1 & 31,83 & 27,05 & 35,13 & 2,54 & $-0,57$ & $-0,02$ & 7,98 \\
\hline & 2 & 34,20 & 25,98 & 52,90 & 7,85 & 1,84 & 4,61 & 22,95 \\
\hline \multirow{2}{*}{$\begin{array}{l}\text { CE } \\
\left(\mathrm{dS} \mathrm{m}^{-1}\right)\end{array}$} & 1 & $10,72^{* *}$ & 2,63 & 23,08 & 7,21 & 0,53 & $-0,74$ & 67,26 \\
\hline & 2 & 2,28 & 1,06 & 3,99 & 0,93 & 0,44 & $-0,29$ & 40,79 \\
\hline \multirow{2}{*}{$\begin{array}{l}\mathrm{Na} \\
\left(\mathrm{meq} \mathrm{L}^{-1}\right)\end{array}$} & 1 & $71,60^{*}$ & 10,15 & 174,55 & 61,16 & 0.76 & $-0,73$ & 85,42 \\
\hline & 2 & 16,02 & 8,17 & 27,07 & 5,11 & 1,00 & 2,83 & 31,90 \\
\hline \multirow{2}{*}{$\begin{array}{l}\text { Ca } \\
(\text { meq L-1) }\end{array}$} & 1 & $29,54^{* *}$ & 13,64 & 53,29 & 17,68 & 0,71 & $-1,72$ & 59,85 \\
\hline & 2 & 6,72 & 3,87 & 10,89 & 2,41 & 0,57 & $-0,86$ & 35,86 \\
\hline \multirow{2}{*}{$\begin{array}{l}\mathrm{Mg} \\
\left(\text { meq L }^{-1}\right)\end{array}$} & 1 & $30,19 * *$ & 7,22 & 74,75 & 22,77 & 0,89 & 0,21 & 75,42 \\
\hline & 2 & 5,34 & 2,06 & 11,75 & 3,47 & 0,86 & $-0,55$ & 64,98 \\
\hline \multirow{2}{*}{ RAS } & 1 & 13,18 & 3,10 & 42,30 & 12,52 & 1,84 & 3,60 & 94,99 \\
\hline & 2 & 6,90 & 4,40 & 12,40 & 2,47 & 1,44 & 2,56 & 35,80 \\
\hline \multirow{2}{*}{$\begin{array}{l}\mathrm{Cl} \\
\left(\mathrm{meq} \mathrm{L} \mathrm{L}^{-1}\right)\end{array}$} & 1 & $38,79^{*}$ & 8,00 & 81,60 & 24,12 & 0,35 & $-0,6$ & 62,18 \\
\hline & 2 & $14,19^{*}$ & 3,00 & 29,00 & 8,88 & 0,43 & $-1,16$ & 62,58 \\
\hline \multirow{2}{*}{$\begin{array}{l}\mathrm{SO}_{4} \\
(\text { meq L-1) }\end{array}$} & 1 & $114,80^{* *}$ & 10,8 & 274,6 & 100,3 & 0,61 & $-1,00$ & 87,37 \\
\hline & 2 & 9,50 & 5,90 & 14,10 & 3,21 & 0,34 & $-1,66$ & 33,79 \\
\hline \multirow{2}{*}{$\begin{array}{l}\% \\
\text { Arcilla }\end{array}$} & 1 & $22,78^{* *}$ & 19,00 & 27,00 & 3,38 & 0,02 & $-1,90$ & 14,84 \\
\hline & 2 & 29,78 & 11,00 & 38,00 & 8,50 & $-1,59$ & 2,37 & 28,54 \\
\hline \multirow{2}{*}{$\begin{array}{l}\% \\
\text { Limo }\end{array}$} & 1 & $47,22^{* *}$ & 45,00 & 49,00 & 1,20 & 0,02 & 1,13 & 2,54 \\
\hline & 2 & 36,78 & 29,00 & 41,00 & 4,32 & $-0,87$ & $-0,18$ & 11,75 \\
\hline \multirow{2}{*}{$\begin{array}{l}\% \\
\text { Arena }\end{array}$} & 1 & 30,00 & 26,00 & 36,00 & 3,74 & 0,30 & 0,30 & 12,47 \\
\hline & 2 & 33,33 & 23,00 & 57,00 & 10,14 & 1,76 & 3,84 & 30,42 \\
\hline
\end{tabular}

Según Anova, promedios con $\left({ }^{*}\right)$ presentan diferencias significativas en el nivel $\infty=0,05$ y $\left(^{* *}\right)$ altamente significativas en el nivel $\infty=0,01$. 
fuente es el Distrito de Riego de la Ramada (alta carga de sólidos en suspensión). En particular, en el lote 1 los contenidos de $\mathrm{Ca}$ y $\mathrm{Mg}$ (elevados en términos absolutos pero con deficiencia de $\mathrm{Ca}$ ) indican una posible interacción de los altos niveles de $\mathrm{Na}$ y el $\mathrm{pH}$ relativamente bajo para un suelo con tendencia a sodicidad (FAO, 1988).

Las diferencias entre los lotes evaluados se confirma en los contenidos de $\mathrm{Cl} \mathrm{y} \mathrm{SO}_{4}$, con variabilidad entre alta y muy alta (exceptuando $\mathrm{SO}_{4}$ en el lote 2), y promedios considerablemente superiores (diferencias altamente significativas) en el lote 1 con respecto al lote 2 . Para la RAS, el lote 1 mostró valores promedio $>13$ (límite para sodicidad), pero debido a la alta variabilidad de esta en ese lote no se presentaron diferencias significativa con el lote 2 . La CIC resultó alta (>20 meq por cada $100 \mathrm{~g}$ ) en los dos lotes, sin diferencias significativas entre estos.

Según la textura, el lote 1 se clasifica como franco y el lote 2 como franco arcilloso con diferencias significativas en términos de arcilla y limo (tabla 4). En conjunto los resultados promedio para el lote 1 muestran una tendencia a un suelo salino-sódico (RAS >13, CE >4, altos contenidos de $\mathrm{Na}, \mathrm{Ca}, \mathrm{Mg}$ y $\mathrm{Cl}_{\text {y SO}}$, pero con $\mathrm{pH}$ $<8,2)$, mientras que el lote $2(2<\mathrm{CE}<4$, RAS $<13$ ) se puede clasificar como lote salino, con altos contenidos de $\mathrm{Cl}$ y SO 4 (FAO, 1988; Soil Survey Staff, 1993).

Los valores de $\mathrm{CE}>4$ en el lote 1 y de $\mathrm{CE}>2$ en el lote 2, particularmente cuando el $\mathrm{Cl}$ es predominante, conllevan a efectos negativos en el rendimiento de cultivos moderadamente tolerantes y sensibles, respectivamente; esta situación se puede ver agravada debido a los desbalances de nutrientes en presencia de $\mathrm{Na}$ (sobre todo en el lote 1) (Grieve et al., 2012).

Los resultados muestran que los dos lotes exigen manejos diferentes. El lote 1 con mayor tendencia a sodicidad requiere enmiendas que desplacen el $\mathrm{Na}$ absorbido en el complejo de cambio, su reemplazo por Ca (deficiente en ese lote) y el lavado del exceso de $\mathrm{Na}$ de la solución del suelo, conservando cierta permeabilidad remanente para que el lavado sea eficiente (García, 2009). Para el lote 2, se deben mejorar las condiciones de drenaje (tabla 1), propiciar el lavado de sales y realizar adiciones de Ca y abonos orgánicos (Ayers y Westcot, 1994; Horneck et al., 2007; Mushtaque et al., 2013). No obstante, la heterogeneidad encontrada requiere un análisis espacial en el interior de cada lote, tal y como se presenta a continuación.

\section{Salinidad y otras propiedades fisicoquímicas del suelo por zonas}

En el lote 1 se encontraron diferencias significativas entre las tres $\mathrm{ZM}$ para $\mathrm{CE}, \mathrm{SO}_{4}$, A y Ar; pero los suelos de las tres ZM se clasificaron texturalmente como francos según United States Department of Agriculture (USDA) (tabla 5). Adicional a estas diferencias, la ZM con "alta" CEa mostró valores significativamente superiores de $\mathrm{pH}, \mathrm{Mg}$, RAS y Cl con respecto a la ZM "baja". Esa ZM "alta" presentó valores considerablemente elevados de CIC, CE, Ca, Mg (la relación $\mathrm{Ca}$ / $\mathrm{Mg}$ indica deficiencia de $\mathrm{Ca}$ ), $\mathrm{RAS}, \mathrm{Cl} \mathrm{y} \mathrm{SO}_{4}$, mostrando que es la zona con mayor tendencia a la sodicidad, incluso con el mayor $\mathrm{pH}$, a pesar de ser inferior al umbral de 8,2 (FAO, 1988). En la zona denominada "media" se encontraron valores elevados de CIC, CE, Ca, Mg (la relación $\mathrm{Ca}$ /

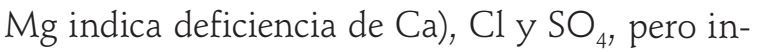
feriores a los reportados en la zona "alta". Por su parte en la zona denominada "baja", exceptuando CIC, se encontraron los menores valores de dichas propiedades; sin embargo, los contenidos de $\mathrm{Cl}_{\text {y SO}}$, se consideran elevados.

El lote 2, con suelos más pesados, pH más bajo y menores contenidos de sales, resultó más homogéneo que el lote 1, pero la ZM con CEa "alta" mostró valores superiores significativamente diferentes de $\mathrm{Cl}$ con respecto a la zona "media" $\mathrm{y}$ de $\mathrm{Ca}, \mathrm{Cl}, \mathrm{Mg}$ con respecto a la ZM "baja". La ZM "alta" presentó valores considerablemente 
elevados de $\mathrm{Ca}, \mathrm{Mg}$ (la relación $\mathrm{Ca} / \mathrm{Mg}$ indica deficiencia de $\mathrm{Ca}$ ), $\mathrm{Cl} \mathrm{y} \mathrm{SO}_{4}$ (tabla 5), mostrando que es la zona más salina (FAO, 1988). Las zonas denominadas "media" y "baja" se diferencian básicamente por los valores de RAS (significativamente mayores en la zona baja) reportando valores elevados de $\mathrm{CIC}, \mathrm{Ca}, \mathrm{Mg}$ (la relación $\mathrm{Ca}$ / $\mathrm{Mg}$ indica deficiencia de $\mathrm{Ca}$ ), $\mathrm{Cl}$ y $\mathrm{SO}_{4}$, pero en general inferiores a los reportados en la zona "alta". En aspectos de textura no se encontraron diferencias significativas entre zonas, pero los contenidos de partículas conllevan a clasificar según USDA como franco arcillosos a los suelos de las zonas "media" y "baja", y como franco limosos a los de la zona "alta".

Los resultados anteriores destacan las ventajas de la identificación de las ZM a partir de la CEa y además confirman que el análisis de esos lotes requiere considerar la heterogeneidad de los contenidos de sales y de la textura entre ZM, lo cual coincide con lo reportado por otros investigadores (Corwin et al., 2003; Lesch et al., 2005). El análisis de resultados requiere considerar las tres vías por las cuales se conduce la corriente en el suelo: i) fase líquida (depende principalmente del Wsc); ii) fase sólida/líquida, con presencia de cationes intercambiables disueltos en el agua, iii) fase sólida, con partículas de suelo en contacto entre sí (Corwin y Lesch, 2005). En esos lotes, el Wsc no mostró mayor correlación espacial con la CEa (prueba de cokriging ya mencionada), pero sí los cationes intercambiables, lo cual destaca la importancia de la fase sólida/líquida. Con respecto a la fase sólida, la relación inversa CEa y Ar en el lote 1 (mayor $\mathrm{CEa}$ en zonas de menor contenido de Ar), contrasta con lo encontrado por otros investigadores (Sudduth et al., 2005), probablemente debido a la predominancia de sales (Corwin et al., 2012).

Los resultados muestran que el manejo de los lotes evaluados debe incluir: selección cuidadosa de cultivos, mejoras en el manejo del riego (verificando la calidad del agua usada), la fertilización (considerando abonos orgánicos) y la labranza. Específicamente en el lote 1, se recomiendan enmiendas tales como yeso (hay deficiencia de $\mathrm{Ca}$ ) o $\mathrm{S}$ en dosis ajustadas a las diferencias encontradas entre ZM, y posteriores lavados. Para el lote 2 se recomiendan aplicaciones de $\mathrm{Ca}$, mejorar condiciones de drenaje y realizar lavado de sales ajustando las láminas e intensidades del riego según las diferencias entre ZM (Ayers y Westcot, 1994; Horneck et al., 2007).

\section{CONCLUSIONES}

El estudio mostró que el sensor de inducción electromagnética EM38-MK2 permitió identificar de manera rápida y confiable diferencias en

Tabla 5. Comparación de medias para las propiedades fisicoquímicas de los dos lotes evaluados.

\begin{tabular}{|c|c|c|c|c|c|c|c|c|c|c|c|c|c|}
\hline Lote & Zona & $\mathrm{pH}$ & $\begin{array}{c}\mathrm{ClC} \\
\mathrm{meq} / 100 \\
\mathrm{~g}\end{array}$ & $\begin{array}{c}\text { CE } \\
\mathrm{dS} \mathrm{m}^{-1}\end{array}$ & $\begin{array}{c}\mathrm{Na} \\
\operatorname{meq} \mathrm{L}^{-1}\end{array}$ & $\begin{array}{c}\text { Ca } \\
\text { meq } L^{-1}\end{array}$ & $\begin{array}{c}\text { Mg } \\
\text { meq L-1 }\end{array}$ & RAS & $\frac{\mathrm{Cl}}{\mathrm{meq} \mathrm{L}^{-1}}$ & $\mathrm{SO}_{4}$ meq & $\mathrm{Ar}$ & $(\%)$ & $A$ \\
\hline \multirow{3}{*}{1} & Baja & $5,5 \mathrm{a}$ & 32,2 a & $3,2 \mathrm{a}$ & $13,4 \mathrm{a}$ & $14,2 \mathrm{ab}$ & 9,0 a & $3,9 \mathrm{a}$ & $14,6 \mathrm{a}$ & $15,7 \mathrm{a}$ & 26,3 a & $47,7 \mathrm{a}$ & $26 a$ \\
\hline & Media & $5,7 \mathrm{ab}$ & $32,4 \mathrm{a}$ & $10,1 \mathrm{~b}$ & $58,1 \mathrm{a}$ & $42,5 \mathrm{a}$ & $35,4 a b$ & $9,5 \mathrm{~b}$ & $43,0 \mathrm{ab}$ & $93,2 \mathrm{~b}$ & $23,0 \mathrm{~b}$ & $47,0 \mathrm{a}$ & $30 \mathrm{~b}$ \\
\hline & Alta & $5,8 \mathrm{~b}$ & 30,9 a & $18,8 \mathrm{c}$ & $143,4 \mathrm{~b}$ & $31,9 \mathrm{~b}$ & $46,1 \mathrm{~b}$ & $26,2 \mathrm{~b}$ & $58,8 \mathrm{~b}$ & 235,6 c & $19,0 \mathrm{c}$ & $47,0 \mathrm{a}$ & $34 \mathrm{c}$ \\
\hline \multirow{3}{*}{2} & Baja & $5,1 \mathrm{a}$ & 36,8 a & $1,9 \mathrm{a}$ & $18,8 \mathrm{a}$ & $5,0 \mathrm{a}$ & 2,6 a & 9,5 a & $10,7 \mathrm{a}$ & $9,4 \mathrm{a}$ & $32,7 \mathrm{a}$ & 38,3 a & $29 \mathrm{a}$ \\
\hline & Media & $5,3 \mathrm{a}$ & $33,6 \mathrm{a}$ & $1,8 \mathrm{ab}$ & $12,1 \mathrm{a}$ & $6,0 \mathrm{ab}$ & $4,9 \mathrm{ab}$ & 5,3 a & $7,6 \mathrm{~b}$ & $10,1 \mathrm{a}$ & $29,0 \mathrm{a}$ & $37,0 \mathrm{a}$ & $34 \mathrm{a}$ \\
\hline & Alta & $5,1 \mathrm{a}$ & 32,2 a & $3,2 \mathrm{~b}$ & $17,2 \mathrm{a}$ & $9,1 \mathrm{~b}$ & $8,5 \mathrm{ab}$ & $5,9 \mathrm{~b}$ & $24,2 \mathrm{c}$ & 9,0 a & $27,7 \mathrm{a}$ & $35,0 \mathrm{a}$ & $37 \mathrm{a}$ \\
\hline
\end{tabular}

Promedios con letras distintas indican diferencia significativa según la prueba DMS $(P \leq 0,05)$. 
propiedades químicas del suelo asociadas a la salinidad (sales y $\mathrm{Na}$ ).

Los patrones espaciales de la CEa permitieron identificar diferencias significativas en propiedades físico-químicas de los suelos al interior de los dos lotes agrícolas estudiados, los cuales difieren entre sí, siendo uno de carácter salino y el otro con tendencia a salino-sódico.

La metodología usada, basada en la determinación de la variabilidad espacial de $\mathrm{CEa}$, permitió identificar en cada lote evaluado distintas ZM, que difieren en las propiedades asociadas a la salinidad y por consiguiente requieren manejo diferenciado por sitio. Esto confirma las bondades de la delimitación de zonas realizada.

Los resultados ilustran el alto potencial del sensor Geonics EM-38MK-2 en la delimitación de ZM con fines de FSE, en razón a su versatilidad y confiabilidad. La metodología usada hace un aporte significativo para definir el manejo por sitio-específico de suelos salinos en la agricultura.

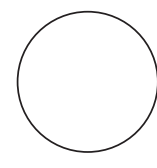

Athar, H. y M. Ashraf. 2009. Strategies for crop improvement against salinity and drought stress: An overview. En: Ashraf, M., M. Ozturk y H.R. Athar (eds.). Salinity and water stress: Improving crop efficiency, tasks for vegetation sciences 44 . Springer, Berlín.

Ayers, R. y D. Westcot. 1994. Water quality for Agriculture. FAO Irrigation and Drainage Paper 29. FAO, Roma.

Benedetto, D., A. Castrignanó, M. Rinaldi, S. Ruggieri, F, Santoro, B. Figorito, S. Gualano, M. Diacono y R. Tamborrino. 2013. An approach for delineating homogeneous zones by using multi-sensor data. Geoderma 199, 117-127. Doi: 10.1016/j.geoderma. 2012.08.028

Burrough, P.A. 1993. Soil variability: a late $20^{\text {th }}$ century view. Soils Fert. 56, 529-562.

Castro, H. y M. Gómez. 2010. Fertilidad de suelos y fertilizantes. pp. 213-303. En: Burbano H. y F. Mojica (eds.). Ciencia del suelo: Principios básicos. Sociedad Colombiana de la Ciencia del Suelo, Bogotá.

Corwin, D., S. Kaffka, J. Hopmans, Y. Mori, J. Van Groeningen y C. Van Kessel. 2003. Assessment and field-scale mapping of soil quality properties of a saline-sodic soil. Geoderma 114(3-4), 231-259. Doi: 10.1016/S0016-7061(03)00043-0

Corwin, D. y S. Lesch. 2005. Characterizing soil spatial variability with apparent soil electrical conductivity I. Survey protocols. Comput. Electron. Agric. 46(1-3), 103-133. Doi: 10.1016/ j.compag.2004.11.002

\section{REFERENCIAS BIBLIOGRÁFICAS}

Corwin, D. y S. Lesch. 2003. Application of soil electrical conductivity to precision agriculture: theory, principles, and guidelines. Agron. J. 95 (3), 455-471. Doi: 10.2134/agronj2003.0455

Corwin, D., S. Lesch y D. Lobell. 2012. Laboratory and field measurements. pp. 295-341. En: Wallender, W.W. y K.K. Tanji (eds.). ASCE Manual and Reports on Engineering Practice No. 71. Agricultural Salinity Assessment and Management. $2^{\text {nd }} e d$. ASCE, Reston, VA.

Corwin, D., S. Lesch, P. Shouse R. Soppe y J. Ayars. 2003. Identifying soil properties that influence cotton yield using soil sampling directed by apparent soil electrical conductivity. Agron. J. 95, 352-364. Doi: 10.2134/agronj2003.0352

Corwin, D. y J. Rhoades. 1982. An improved technique for determining soil electrical conductivity - depth relations from above ground electromagnetic induction measurements. Soil Sci. Soc. Am. J. 46, 517-520. Doi: 10.2136/sssaj1982.03615995004600030014x

Doolittle, J. y E. Brevik. 2014. The use of electromagnetic induction techniques in soils studies. Geoderma223-225,33-45.Doi:10.1016/j.geoderma. 2014.01.027

ESRI. 2011. ArcGIS Desktop: Release 10. Environmental Systems Research Institute, Redlands, CA.

FAO. 1988. Salt-affected soils and their management. FAO Soils Bull. 39, 131.

García, A. 2009. Manejo de suelos con acumulación de sales. pp. 8-10. En: Memorias VIII Congreso Ecuato- 
riano de la Ciencia del Suelos, Sociedad Ecuatoriana de la Ciencia del Suelo, Esmeraldas, Ecuador.

Horneck, D.A., J.W. Ellsworth, B.G. Hopkins, D.M. Sullivan y R.G. Stevens. 2007. Managing saltaffected soils for crop production. PNW 601-E. Extension Service, Oregon State University, Corvallis, OR.

IBM. 2013. Corp. Released, IBM Stadistics for Windows, Version 22.0. IBM Corp., Armonk, NY.

Instituto Geográfico Agustín Codazzi (IGAC). 2014. Levantamiento agrológico del Centro Agropecuario Marengo (CAM). Subdirección de Agrología, Universidad Nacional de Colombia, Bogotá.

Larreal, R. 2005. Definición y establecimiento de la serie San Francisco en la altiplanicie de Maracaibo, sector semiárido. Facultad de Agronomía, LUZ, Maracaibo, Venezuela.

Lesch, S., D. Corwin y D. Robinson. 2005. Apparent soil electrical conductivity mapping as an agricultural management tool in arid zone soils. Comp. Electron. Agric. 46, 351-378. Doi: 10.1016/ j.compag.2004.11.007

MADS. 2013. Política Nacional para la Gestión Integral Ambiental del Suelo (GIAS). Ministerio de Ambiente y Desarrollo Sostenible, Bogotá.

Mulla, D. y J. Schepers. 1997. Key process and properties for site-specific soil and crop management. pp. 1-18. En: Pierce, F.J. y E.J. Saldler (eds.). States of Site Specific Management, Madison, WI.

Munns, R. 2009. Strategies for crop improvement in saline soils. pp. 99-110. En: Ashraf, M., R. Ozturk y H.R. Athar (eds.). Salinity and water stress. Improving crop eficiency: Tasks for vegetation sciences 44. Springer, Berlín.

Mushtaque, A., N. Hussain y S. Al-Rawahy. 2013. Management of saline lands in Oman: Learning to live with salinity. pp. 285-291. En: Shahid, S.M. (ed). Developments in soil salinity as- sessment and reclamation: Innovative thinking. Springer, Berlín.

Pautasso, J., S. Albarenque, G. Schulz y A. Kemerer. 2010. Delimitación de zonas de manejo mediante variables de suelo y validación para maíz. Curso Internacional de Agricultura de Precisión. INTA Manfredi, Córdoba, Argentina.

Pla, I. 2014. Nuevas experiencias en la evaluación y diagnóstico de procesos de salinización y sodificación de suelos en América Latina. Suelos Ecuatoriales 44(2), 125-137.

Schepers, J., M. Schlemmer y R. Fergunson. 2000. Site specific considerations form managing phosphorus. J. Environ. Qual. 29,125-130. Doi: 10.2134/ jeq2000.00472425002900010016x

Simón, M., N. Peralta y J. Costa. 2013. Relación entre la conductividad eléctrica aparente con propiedades del suelo y nutrientes. Sociedad de la Ciencia del Suelo, Buenos Aires.

Soil Survey Staff. 1993. Soil survey manual. Handbook 18. USDA, Washington, DC.

Sudduth, K., S. Drummond, S. Birrel y N. Kitchen. 1997. Spatial modeling of crop yield using soil and topographic data. pp. 439-447. En: Strafford, J.V. (ed.). Precision Agriculture '97: Proceedings of the First European Conference on Precision Agriculture. BIOS Scientific Publishers, Oxford, UK.

Sudduth, K., N. Kitchen, G. Bollero, W. Wiebold, W. Batchelor, D., Bullock, D.E. Clay, H.L. Palm, F.J Pierce, R.T. Schuler y K.D. Thelene. 2005. Relating apparent electrical conductivity to soil properties across the north-central USA. Comp. Electron. Agric. 46, 263-283. Doi: 10.1016/j.compag.2004.11.010

Zhaoyong, Z., J. Abuduwaili y H. Yimit. 2014. The occurrence, sources and spatial characteristics of soil salt and assessment of soil salinization risk in Yanqi Basin, Northwest China. Plos One 9(9), 1-12. Doi: 10.1371/journal.pone.0106079 